\title{
Immediate effect of basic body awareness therapy on heart rate variability
}

\author{
Alessandra Madia Mantovani a , Cristina Elena Prado Teles Fregonesi ${ }^{\mathrm{b}}$, \\ Roselene Modolo Regueiro Lorençoni ${ }^{\mathrm{b}}$, Nathalia Ulices Savian ${ }^{\mathrm{b}}$, \\ Mariana Romanholi Palma ${ }^{\mathrm{b}}$, Afonso Shiguemi Inoue Salgado ${ }^{\mathrm{c}}$, \\ Luís Vicente Franco de Oliveira ${ }^{\mathrm{d}}$, Rodolfo Borges Parreira ${ }^{\mathrm{c}, *}$ \\ a PhD Student in Science Motricity, Institute of Biosciences, Universidade Estadual Paulista, Rio Claro, SP, 19060-900, Brazil \\ ${ }^{\mathrm{b}}$ Department of Post Graduate Program of Physical Therapy, Universidade Estadual Paulista, Presidente Prudente, 19060-900 Brazil \\ c School of Postural and Manual Therapy, Salgado Institute of Integral Health, Londrina, 86055-240, PR, Brazil \\ ${ }^{\mathrm{d}}$ Rehabilitation Sciences Master and Doctoral Degree Program - Nove de Julho University, UNINOVE, Sao Paulo, SP, 01504-000, Brazil
}

\section{A R T I C L E I N F O}

Article history:

Received 15 September 2015

Received in revised form

19 October 2015

Accepted 23 October 2015

\section{Keywords:}

Heart rate variability

Autonomic nervous system

Physical therapy

Basic body awareness therapy

\begin{abstract}
A B S T R A C T
Objective: To determine the immediate effect of a Basic Body Awareness Therapy (BAT) session on measures of heart rate variability (HRV) in healthy young people.

Methods: 13 healthy young subjects of both genders, who showed no illnesses related to the autonomic nervous system (ANS) underwent an ANS evaluation before and after conducting a session of BAT. The assessment of ANS activity was conducted through the HR with the aid of Nerve-Express ${ }^{\circledR}$ software. The BAT session lasted for $50 \mathrm{~min}$ and was performed by one investigator (blinded to the assessment procedures).

Results: After BAT session significant improvement was found in the sympathetic and parasympathetic modulation $(p<0.05)$, and the general estimate of heart rate variability $(p<0.04)$.

Conclusion: BAT was found to be an effective, easy to apply and inexpensive therapeutic technique, able to change ANS in order to improve HR which may suggest better health conditions for participating individuals.
\end{abstract}

() 2015 Elsevier Ltd. All rights reserved.

\section{Introduction}

Like other organs of the human body, the heart is dually innervated, and through the wide variety of physiological factors which determine cardiac function (heart rate (HR)), the autonomic nervous system (ANS) is the most relevant, so that a relative increase in sympathetic activity is associated with increased HR while an increase in parasympathetic activity is associated with decreased HR [1]. One method of evaluating a complex system is through temporal analysis of a suitable variable, for example, the

\footnotetext{
* Corresponding author. Rubens Carlos de Jesus, st. \# 355, 86055-240, Londrina, Paraná, Brazil.

E-mail addresses: leka_indy@hotmail.com (A.M. Mantovani), cristina@fct.unesp. br (C.E.P.T. Fregonesi), roselene@fct.unesp.br (R.M.R. Lorençoni), natysavian@ hotmail.com (N.U. Savian), marianaromanholi@hotmail.com (M.R. Palma), afonsosisalgado@yahoo.com.br (A.S.I. Salgado), oliveira.lvf@uninove.br (L.V.F. de Oliveira),dolfo23@yahoo.com (R.B. Parreira).
}

interval between successive beats of a heart cycle [2]. This system is regularly assessed through heart rate variability (HRV), using markers of differentiation of the heart, which allows continuous observations of fluctuations in heart rate related to the sympathetic and parasympathetic efferent activities, directed to the sinus node and "fired" by the central and peripheral receptors [3,4].

$\mathrm{HRV}$ is analyzed in the time and frequency domain analysis where the time variations are quantified as the differences between successive RR intervals [3], while the analysis in the frequency domain allows the calculation of high frequency, which is mediated by vagal activity [5], and low frequency, which has been suggested to represent predominantly sympathetic modulation [6]. This technique is widespread among various fields of physiotherapy for the evaluation and promotion of mental or physical welfare [7-11]. In the context of physical therapy, Basic Body Awareness Therapy (BAT) is a mind-body activity tool that focuses on body awareness as a basic level of self-awareness [12].

Basic Body Awareness Therapy (BAT) is a form of physical 
therapy with a holistic approach that involves focusing attention on awareness of internal body sensations, directed to an awareness of how the body is used in terms of behavior, interaction with the self and others [12,13]. Basic BAT consists of simple exercises such as stopping moving whilst sitting, lying, standing or walking, with an emphasis on each moment of the movement. The principles used in this therapy are to work with experiences to increase contact with the ground, postural control and awareness of the body [12]. A focus on emotions is important in basic BAT; it helps patients to become aware of feelings and emotions in the body, one of the main foci of treatment, and the physiotherapist confirms these emotions as they are continually emerging [14]. It has been seen that psychological factors such as anxiety and depression and coping strategies are important predictors in the genesis of chronic musculoskeletal conditions [15].

Basic BAT can be used in various clinical settings both for prevention and rehabilitation, particularly by improving aspects of pain and mental health as demonstrated above, however, to analyze the relationship between basic BAT and the body-mind integration and modulation of emotions on the behavior of the ANS, it is necessary to investigate the possible changes through HRV analysis before a session of basic BAT. The aim of this study was to determine the immediate effect of a session of basic BAT on the HRV parameters of healthy young people.

\section{Methods}

\subsection{Participants}

The sample consisted of 13 subjects, two males and 11 females (20.5 \pm 0.76 yrs), which of the total participants, $85 \%$ regularly engaged in physical activity. Subjects are from the student body of the Faculty of Science and Technology, Universidade Estadual Paulista, Presidente Prudente/SP-Brazil, who presented no illnesses related to the ANS. Participants were asked to refrain from stimulants and/or alcoholic substances for at least $12 \mathrm{~h}$ prior to the ANS examination. All procedures were submitted to and approved by the Research Ethics Committee of FCT/UNESP (Protocol $\mathrm{n}^{0}$ 2178113.9.0000.5402) and all participants, after reading and agreeing to the procedures and purposes of the study, signed a Free and Clear Consent Form.

\subsection{Assessment}

Initially, personal data was collected through a semi-structured interview, following which; the participants underwent an anthropometric assessment (weight, height and body mass index). The ANS activity (sympathetic and parasympathetic), through HRV, was evaluated both before and after a session of basic BAT, with the aid of Nerve-Express ${ }^{\mathbb{B}}$ software (Heart Rhythm Instruments, Metuchen, NJ, EUA). Data were recorded by means of a transmitter belt (Polar ${ }^{\circledR}$ T31 coded ${ }^{\mathrm{TM}}$ transmitter, Electro Oi, Finland), placed on the chest of the participant at the xiphoid process, and a heart rate receiver attached to the waist and linked to a computer for processing and storing captured data.

The subject began the test on a stretcher in the supine position and after 192 RR intervals the patient was asked to stand up and looking at a wall, remaining in this position for a few minutes until the end of the test. The test was determined as being completed after $448 \mathrm{RR}$ interval points, which represents approximately five minutes. A recording of approximately $1 \mathrm{~min}$ is needed to assess the HF components of HRV while approximately 2 min are needed to address the LF component. In order to standardize the procedure, 5 min recordings were performed according to the standard measurement of heart rate variability of the European Society of
Cardiology and The North American Society of Pacing and Electrophysiology (Task Force of the European Society of Cardiology and the North American Society of Pacing and Electrophysiology 1996).

All assessments took place in a controlled environment (i. e. light, temperature, and sound). HRV signals were compiled with MATLAB routines (The Mathworks, Natick, MA) and analyzed in both the time and frequency domain using the software Kubios HRV version 1.1 (Biosignal Analysis and Medical Imaging Group; Kuopio, Finland). The frequency bands analyzed in this study were in accordance with previous guidelines published by the American Heart Association which state the recommendations for HRV measurement and interpretation [16]. The variables used in this study for the time domain (obtained by determination of correspondent RR intervals at any point in the time) [16,17], were mean $\mathrm{RR}$ (mean values of $\mathrm{N}-\mathrm{N}$ intervals from a period of time), HR intervals (beats/min), SDNN (standard deviation of the $\mathrm{N}-\mathrm{N}$ intervals - an estimate of total HRV) and rMSSD (square root of the mean squared difference of the successive $\mathrm{N}-\mathrm{N}$ intervals $-\mathrm{a}$ parasympathetic marker); these variables are routinely measured in the supine position. In the time domain a nonlinear method, the Poincare plot, was used, denoted by SD1 that describes the standard deviation of the beat-to-beat short-term variability, characterized as a marker of parasympathetic modulation; and SD2 which represents the long-term standard deviation of the continuous $\mathrm{R}-\mathrm{R}$ intervals, characterized as a marker of parasympathetic and sympathetic modulation [18]. HRV triangular index (HRVtri) was obtained as the total number of RR intervals divided by the height of the histogram which depends on the selected bin width [16]. This method present RR intervals in geometric patterns and various approaches are used to derive measures of HRV from them, and is an estimate of overall heart rate variability [19].

The frequency domain (contributing to an understanding of the autonomic background of RR interval fluctuations in the recorded heart rate) was measured in both the supine and orthostatic positions, and the variables analyzed were; the low frequency power (LF - is modulated by both parasympathetic and sympathetic activities) and high frequency power ( $\mathrm{HF}$ - modulated exclusively by parasympathetic activities). The LF/HF ratio was considered as a marker of the sympathovagal balance.

\subsection{Therapeutic intervention}

The body awareness performed in this study it was based on body working methods devised by physiotherapist Serge Peyrot (creator of the Morfoanalitica Therapy) [20,21,22]. The session was led by a physical therapist with background in Morfoanalitica Therapy. The BAT session lasted for $50 \mathrm{~min}$ in a comfortable environment, regarding light and sound. The practice included body awareness, moving, diaphragmatic breathing and massage. The session began with participants standing. Later they were in the supine position and, as guidelines were getting consciousness touches and non-touches the body structures with the ground. They were oriented to the perception of diaphragmatic breathing. The massage was made with the body movement on a foam cervical support or body movement on the ground. The session ended with the participant feeling again each body part and realizing how responded to therapy. After was performed a group moment, where each participant shared the bodily sensations and feelings experienced during therapy. The experience of body awareness began asking to the participant to feel the different parts of the body - through the own presence of the body part to perceptions of the sensations of the skin surface, weight and volume, so get in touch with your body [23]. 


\subsection{Data analyzes}

All data were analyzed by a subject who was not engaged in this study, which was blinded in relation to the status of the participants. All analysis was performed using the statistical program Graph Pad Prism ${ }^{\circledR}$ version 5.00 for Windows (GraphPad Software, San Diego, California, USA). Personal data such as weight, height and BMI were expressed by descriptive statistics with mean and standard deviation. The normality of the HRV parameters was tested using the Shapiro-Wilk test and comparison of the moments before and after the intervention using the paired t test. A significance level of $5 \%$ was adopted.

\section{Results}

Tables 1 and 2 describe the linear and nonlinear indices of heart rate variability before and after application of basic body awareness therapy. Table 1 shows that there was a statistical difference ( $p<0.05$ ), before and after basic BAT, only in the HRV component that is the sympathetic and parasympathetic modulation (SD2). The parameter that reflects the overall behavior in both the shortand long-term (SDNN) almost reached statistical significance $(\mathrm{p}=0.06)$, however, the general estimate of heart rate variability over time (HRVtri) showed statistical significance $(\mathrm{p}=0.04)$.

With regard to the components in the frequency domain, only the parameter that represents the low frequencies (LF) showed a statistically significant difference $(p<0.05)$, after application of basic BAT (Table 2).

\section{Discussion}

This study examined the immediate effect of a session of basic body awareness therapy on heart rate variability in healthy young people, through linear and non-linear indices of HRV. The results showed a significant increase in the LF and SD2 variables suggesting that immediately following a session of basic BAT there is an improvement in the functioning of autonomic control with modulation of both the vagal and sympathetic systems.

Basic BAT has been favorably compared with other forms of therapy for pain conditions and psychological symptoms in patients with chronic musculoskeletal disorders [23]. Eriksson et al. [24] showed that the effects of basic BAT persisted for up to 6 months with alleviation of gastrointestinal and psychological symptoms, such as abdominal pain, tension and improved body awareness in patients with irritable bladder syndrome. Other aspects of basic BAT have also had positive effects. Mannerkorpi and Gard [25] analyzed patients who participated in a physical therapy group with exercises in the pool including some aspects of body awareness and found that these patients had a more positive perception of their body, were more relaxed and had developed a

Table 1

Components of heart rate variability in the time domain.

\begin{tabular}{llll}
\hline Variable & Before mean $(\mathrm{SD})$ & After mean $(\mathrm{SD})$ & $p$-value \\
\hline rMSSD & $23.91(20.7)$ & $30.16(19.3)$ & 0.21 \\
SDNN & $26.95(12.6)$ & $40.31(28.8)$ & 0.06 \\
SD1 & $16.92(14.7)$ & $27.36(28.2)$ & 0.21 \\
SD2 & $32.80(14.3)$ & $48.82(30.1)$ & $\mathbf{0 . 0 3}^{*}$ \\
HRVtri & $2.277(1,48)$ & $3.218(2,29)$ & $\mathbf{0 . 0 4}^{*}$ \\
\hline
\end{tabular}

* statistical difference $\mathrm{p}<0.05$

$\mathrm{SD}$, standard deviation; rMSSD, square root of the mean squared difference of the successive $\mathrm{N}-\mathrm{N}$ intervals; SDNN, standard deviation of $\mathrm{N}-\mathrm{N}$ intervals; SD1, standard deviation of the beat-to-beat instantaneous variability; SD2, long-term standard deviation of continuous $\mathrm{R}-\mathrm{R}$ intervals; SD1/SD2, long-term standard deviation of continuous $\mathrm{R}-\mathrm{R}$ intervals; HRVtri, estimate of overall heart rate variability.
Table 2

Components of heart rate variability in the frequency domain.

\begin{tabular}{llll}
\hline Variable & Before mean (SD) & After mean (SD) & $p$-value \\
\hline HFnu & $466.4(197)$ & $223.2(168)$ & 0.49 \\
LFnu & $353.5(293)$ & $881.1(111)$ & $\mathbf{0 . 0 2}^{\star}$ \\
LF/HF & $1.696(1.18)$ & $3.052(2.41)$ & 0.20 \\
\hline
\end{tabular}

* statistical difference $\mathrm{p}<0.05$

$\mathrm{SD}$, standard deviation; HFnu, high frequency in normalized units; LFnu, low frequency in normalized units; LF/HF, ratio between LF and HF band powers.

more trusting and tolerant relationship between their body and self. This process was independent of pain or whether the condition had improved or remained unchanged. It was also shown that after a period of treatment with basic BAT, the salivary cortisol slope measured during the day was normalized [24]. The salivary cortisol slope has been reported as an indicator of cumulative physiological and psychological stress [26].

Stress factors have been reported with low parameters in parasympathetic activity and a low dominance of sympathetic over parasympathetic. The increase in the $\operatorname{LF}(\mathrm{p}=0.02)$ index with a concomitant elevation in the SDNN (Table 1) and SD2 parameter and no significant changes in the LF/HF ratio (Table 2), can be interpreted as a positive effect of therapy, thus suggesting that basic BAT promoted a better balance between the sympathetic and parasympathetic action of the individuals tested. It is known that the LF index (mainly expressed in normalized units) shows activity that is typically modulated by the sympathetic action of the ANS, however, it also represents parasympathetic action, while the HF index has full parasympathetic predominance, and the ratio between the two indices produces information about the sympathetic and parasympathetic balance of the system [17,27]. To date, no related studies have been found showing the effect of basic BAT on the autonomic nervous system, however, these findings agree with a study by Díaz-Rodrígues et al. [28] where the effects of the Reiki technique on ANS were analyzed in healthy subjects and verified for the same purposes as the present study, with a shift in the autonomic balance toward parasympathetic predominance.

The results of this study support the hypothesis that basic BAT can modulate the autonomic balance and rhythm as it was determined by the sympathetic and parasympathetic inputs to the heart and this improves the balance, as demonstrated by the SDNN, SD2, HRVtri and LF indices. The RRtri and SD2 indices, characteristic of autonomic modulation global1, were significantly increased after the session of basic BAT (Tables 1 and 2), suggesting an increase in the overall variability of HR [25]. Such findings corroborate research using other mind-body techniques such as Reiki [28], Yoga [29], manual therapy [30] and meditation [31] which after the intervention, showed an increase in perceived well-being accompanied by an increase in HRV, suggesting a positive relationship between welfare, the cardioprotective effect and associated breathing techniques, present in all the described techniques including basic BAT favoring the equilibrium of ANS [31]. Thus, it is likely that this technique helps in balancing the ANS as well as improving the physical and emotional aspects of individuals.

There are some limitations to consider. The first is related to sample size, as, due to a lack of related studies, we used a convenience sample, pairing by sex would be interesting to balance the results, however, a study by Ramaerkers et al. [3] showed no gender difference in the cardiac autonomic function of adults. Secondly, the generalizability of the findings may be limited due to the fact that the technique was applied by a specialist physiotherapist, and only in healthy subjects in whom it would be interesting conduct this intervention in patients with comorbidities; so the results should be extrapolated with caution. Finally, the immediate analysis of HRV could limit the clinical significance of the data, as 
opposed to a long-term analysis where the researcher should consider the use of a 24-h Holter. It is worth pointing out the fact that, to date, no studies analyzing the effects of techniques of Basic Body Awareness Therapy on heart rate variability have been found.

\section{Conclusion}

We can concluded that Basic Body Awareness Therapy was able to induce adjustments in the autonomic modulation of heart rate variability, indicating good physiological functioning as seen in some parameters such as HRVtri, SD2, and LF, thereby showing a possible balance of autonomic nervous system.

\section{Conflict of interest statement}

The author declare have no conflicts of interest related to this work.

\section{References}

[1] J.F. Thayer, F. Ahs, M. Fredrikson, J.J. Sollers 3rd, T.D. Wager, A meta-analysis of heart rate variability and neuroimaging studies: implications for heart rate variability as a marker of stress and health, Neurosci. Biobehav Rev. 36 (2) (2012) 747-756.

[2] A.J. Seely, P.T. Macklem, Complex systems and the technology of variability analysis, Crit. Care 8 (6) (2004) R367-R384.

[3] D. Ramaekers, H. Ector, A.E. Aubert, A. Rubens, F. Van de Werf, Heart rate variability and heart rate in healthy volunteers. Is the female autonomic nervous system cardioprotective? Eur. Heart J. 19 (9) (1998) 1334-1341.

[4] A.J. Rassias, P.M. Guyre, M.P. Yeager, Hydrocortisone at stress-associated concentrations helps maintain human heart rate variability during subsequent endotoxin challenge, J. Crit. Care 26(6) (636) (2011) e1-5.

[5] J. Hayano, Y. Skakibara, A. Yamada, M. Yamada, S. Mukai, T. Fujinami, et al, Accuracy of assessment of cardiac vagal tone by heart rate variability in normal subjects, Am. J. Cardiol. 67 (2) (1991) 199-204.

[6] B. Pomeranz, R.J.B. Macaulay, M.A. Caudill, I. Kutz, D. Adam, D. Gordon, et al. Assessment of autonomic function in humans by heart rate spectral analysis, Am. J. Physiol. 248 (1Pt 2) (1985) H151-H153.

[7] J.P. Delaney, K.S. Leong, A. Watkins, D. Brodie, The short-term effects of myofascial trigger point massage therapy on cardiac autonomic tone in healthy subjects, J. Adv. Nurs. 37 (4) (2002) 364-371.

[8] L.Y.A. Kawaguchi, A.C.P. Nascimento, M.S. Lima, L. Frigo, A.R.P. Júnior, C.J. Tierra-Criollo, R.A.B. Lopes-Martins, Characterization of heart rate variability and baroreflex sensitivity in sedentary individuals and male athletes, Rev. Bras. Med. Esporte 13 (4) (2007) 207e-212e.

[9] S. Boettger, D. Hoyer, K. Falkenhahn, M. Kaatz, V.K. Yeragani, K.J. Bär, Nonlinear broad band dynamics are less complex in major depression, Bipolar Disord. 10 (2) (2008) 276-284.

[10] R. Köbele, M. Koschke, S. Schulz, G. Wagner, S. Yeragani, C.T. Ramachandraiah, et al., The influence of negative mood on heart rate complexity measures and baroreflex sensitivity in healthy subjects, Indian J. Psychiatry 52 (1) (2010) $42-47$.

[11] C.A. Camillo, M. Laburu Vde, N.S. Gonçalves, V. Cavalheri, F.P. Tomasi, N.A. Hernandes, et al., Improvement of heart rate variability after exercise training and its predictors in COPD, Respir. Med. 105 (7) (2011) 1054-1062.

[12] L. Hedlund, A.L. Gyllensten, The physiotherapists' experience of basic body awareness therapy in patients with schizophrenia and schizophrenia spectrum disorders, J. Bodyw. Mov. Ther. 17 (2) (2013) 169-176

[13] W.E. Mehling. J. Wrubel, JJ. Daubenmier, C.J. Price, C.E. Kerr, T. Silow, et al., Body awareness: a phenomenological inquiry into the common ground of mind-body therapies, Philos. Ethics Humanit Med. 6 (2011) 6.

[14] G. Gard, A. Lundvik-Gyllensten, The importance of emotions in physiotherapeutic practice, Phys. Ther. Rev. 5 (3) (2000) 155-160.

[15] E.B. Malmgren-Olsson, B.A. Armelius, K. Armelius, A comparative outcome study of body awareness therapy, feldenkrais, and conventional physiotherapy for patients with nonspecic musculoskeletal disorders: changes in psychological symptoms, pain, and self-image, Phys. Ther. Pract. 17 (2) (2001) $77-95$.

[16] M. Malik, J.T. Bigger, A.J. Camm, R.E. Kleiger, A. Malliani, A.J. Moss, et al., Heart rate variability: standards of measurement, physiological interpretation, and clinical use, Eur. Heart J. 17 (3) (1996) 354-381.

[17] L.C.M. Vanderley, C.M. Pastre, R.A. Hoshi, T.D. Carvalho, M.F. Godoy, Basic notions of heart rate variability and its clinical applicability, Rev. Bras. Cir. Cardiovasc 24 (2) (2009) 205-217.

[18] L. Mourot, M. Bouhaddi, S. Perrey, J.D. Rouillon, J. Regnard, Quantitative poincaré plot analysis of heart rate variability: effect of endurance training, Eur. J. Appl. Physiol. 91 (1) (2004) 79-87.

[19] U. Rajandra Acharya, K. Paul Joseph, N. Kannathal, C.M. Lim, J.S. Suri, Heart rate variability: a review, Med. Biol. Eng. Comput. 44 (12) (2006) 1031-1051.

[20] R.W. Johnsen, M. Råheim, Feeling more in balance and grounded in one' s own body and life. Focus group interviews on experiences with Basic Body Awareness Therapy in psychiatric healthcare, Adv. Physiother. 12 (3) (2010) $166-174$.

[21] D. Catalan-Matamoros, L. Helvik-Skjaerven, M.T. Labajos-Manzanares, A. Martínez-de-Salazar-Arboleas, E. Sánchez-Guerrero, A pilot study on the effect of basic body awareness therapy in patients with eating disorders: a randomized controlled trial, Clin. Rehabil. 25 (7) (2011) 617-626.

[22] A. Lundvik-Gyllensten, L. Hansson, C. Ekdahl, Patient experiences of basic body awareness therapy and the relationship with the physiotherapist, J. Bodyw. Mov. Ther. 7 (3) (2003) 173-183.

[23] G. Gard, Body awareness therapy for patients with fibromyalgia and chronic pain, Disabil. Rehabil. 27 (12) (2005) 725-728.

[24] E.M. Eriksson, I.E. Möller, R.H. Söderberg, H.T. Eriksson, G.K. Kurlberg, Body awareness therapy: a new strategy for relief of symptoms of irritable bowel syndrome patients, World J. Gastroenterol. 13 (23) (2007) 3206-3214.

[25] K. Mannerkorpi, G. Gard, Physiotherapy group treatment for patients with fibromyalgia - an embodied learning process, Disabil. Rehabil. 25 (24) (2003) $1372-1380$.

[26] S.E. Sephton, R.M. Sapolsky, H.C. Kraemer, D. Spiegel, Diurnal cortisol rhythm as a predictor of breast cancer survival, J. Natl. Cancer Inst. 92 (12) (2000) 994-1000.

[27] S. Akselrod, D. Gordon, F.A. Ubel, D.C. Shannon, A.C. Berger, R.J. Cohen, Power spectrum analysis of heart rate fluctuation: a quantitative probe of beat to beat cardiovascular control, Science 213 (4504) (1981) 220-222.

[28] L. Días-Rodrígues, M. Arroyo-Morales, C. Fernández-de-las-Peñas, F. GarcíaLafuente, C. García-Royo, I. Tomás-Rojas, Immediate effects of reiki on heart rate variability, cortisol levels, and body temperature in health care professionals with burnout, Biol. Res. Nurs. 13 (4) (2011) 376-382.

[29] B.H. Krishna, P. Pal, G.K. P, J. B, E. J, Y. S, M.G. S, S.G. G, Effect of yoga therapy on heart rate, blood pressure and cardiac autonomic function in heart failure, J. Clin. Diagn. Res. 8 (1) (2014) 14-16.

[30] C. Toro-Velasco, M. Arroyo-Morales, C. Fernández-de-las-Peñas, J.A. Cleland, F.J. Barrero-Hernández, Short-term effects of manual therapy on heart rate variability, mood state, and pressure pain sensitivity in patients with chronic tension-type headache: a pilot study, J. Manip. Physiol. Ther. 32 (7) (2009) $527-535$.

[31] J.R. Krygier, J.A. Heathers, S. Shahrestani, M. Abbott, J.J. Gross, A.H. Kemp, Mindfulness meditation, well-being, and heart rate variability: a preliminary investigation into the impact of intensive vipassana meditation, Int. J. Psychophysiol. 89 (3) (2013) 305-313. 